\title{
RESEARCH PAPERS
}

Acta Cryst. (1998). B54, 507-514

\section{Correlation of Internal Torsional Motion with Overall Molecular Motion in Crystals $\dagger$}

\author{
Verner SCHOMAKer ${ }^{a} \ddagger$ AND Kenneth N. Trueblood ${ }^{b} \S$ \\ ${ }^{a}$ Beckman Institute, Division of Chemistry and Chemical Engineering, California Institute of Technology, Pasadena, \\ CA 91125, USA and Department of Chemistry, University of Washington, Seattle WA 98195, USA, and ${ }^{b}$ Department \\ of Chemistry and Biochemistry, University of California, Los Angeles, CA 90095-1569, USA
}

(Received 23 December 1997; accepted 23 February 1998)

\begin{abstract}
The simple one-parameter Dunitz-White model for internal torsional motion accompanying overall molecular motion in crystals ignores the correlations between this torsion and the overall translation and libration. These correlations are explicitly considered here, in quadratic approximation (based on the linear approximation for displacements). For each attached rigid group (ARG) undergoing a torsional libration, there are, in addition to the mean-square libration amplitude, six correlations to be considered, three with the overall molecular libration and three with the overall translation. Because it is impossible from the observed quadratic mean displacements to distinguish the torsional motion from the overall molecular libration parallel to the torsional axis, the present analysis emphasizes the overall libration parallel to the torsional axis, which we term $\Lambda$. In the general case there are only six determinable parameters for each ARG. If the torsional axis of the ARG lies on a molecular symmetry element, the number of parameters is reduced to three or fewer. Examples of analyses with these correlations included, and without them, are compared.
\end{abstract}

\section{Introduction}

The assumption that a molecule or atomic grouping behaves as a rigid body in a crystal is the simplest way of introducing amplitude and phase relations among the motions of individual atoms. The rigid-body model has been applied to the analysis of atomic anisotropic

\footnotetext{
$\dagger$ A preliminary account of some of this material was presented at the Hamburg Congress of the IUCr (Schomaker \& Trueblood, 1984), and some has also been presented by Dunitz, Schomaker \& Trueblood (1988) and by Dunitz, Maverick \& Trueblood (1988). In each of those accounts, however, we sometimes alluded to the r.m.s. (root mean square) torsional amplitude $\varphi$ in ways that led some to infer that it might be determinable from the quadratic mean displacements. We try to avoid that here.

‡ Died 30 March 1997

$\S$ Died 7 May 1998.

C) 1998 International Union of Crystallography

Printed in Great Britain - all rights reserved
}

Gaussian displacement parameters (ADP's) from thousands of crystal structures in the four decades since it was first proposed (Cruickshank, 1956), indeed, to many more structures than might reasonably be expected to contain rigid molecules or ions. In general, three tensors are needed, $\mathbf{T}, \mathbf{L}$ and $\mathbf{S}$, to describe the motion of a rigid molecule in a crystal [Schomaker \& Trueblood, 1968; hereafter ST(1968)]. This follows simply on writing

$$
\mathbf{u}=\mathbf{t}+\lambda \times \mathbf{r}
$$

for the infinitesimal displacement of an atom at restposition $\mathbf{r}$ in a rigid molecule when the molecule translates by the vector distance $\mathbf{t}$ and rotates by the angle $\lambda$ about an axis through the origin and parallel to $\lambda$, forming the dyad product

$$
\mathbf{u u}=\mathbf{t t}+\mathbf{t} \lambda \times \mathbf{r}-\mathbf{r} \times \lambda \mathbf{t}-\mathbf{r} \times \lambda \lambda \times \mathbf{r},
$$

and time-and-lattice averaging over all the countless instantaneous displacements of the rigid body

$$
\mathbf{U} \stackrel{\text { def }}{=}\langle\mathbf{u u}\rangle=\mathbf{T}+\mathbf{S}^{\mathbf{T}} \times \mathbf{r}-\mathbf{r} \times \mathbf{S}-\mathbf{r} \times \mathbf{L} \times \mathbf{r},
$$

with $\mathbf{T} \stackrel{\text { def }}{=}\langle\mathbf{t t}\rangle, \mathbf{L} \stackrel{\text { def }}{=}\langle\lambda \lambda\rangle$ and $\mathbf{S} \stackrel{\text { def }}{=}\langle\lambda \mathbf{t}\rangle . \quad \mathbf{L}$ is originindependent; $\mathbf{T}$ and $\mathbf{S}$ change in predictable ways with a change of origin [ST(1968)]. Whereas $\mathbf{T}$ and $\mathbf{L}$ are symmetric second-rank tensors, $\mathbf{S}$ is generally not symmetric. Furthermore, the ADP's cannot determine the diagonal elements of $\mathbf{S}$ completely $\left(\operatorname{Tr}\{S\}=S_{i i}\right.$ is ADP-indeterminate), so that only their differences can be obtained $\dagger \dagger$ (usually the sum of the diagonal elements is arbitrarily set to zero). In the general case, therefore, 20 independent tensor elements $(6+6+8)$ can be determined from the ADP's to describe the rigid-

\footnotetext{
- Equation (1) is the quadratic approximation to the exact expression, discussed in detail in ST(1968).

†† Bürgi (1995) and Förtsch (1997) have recently shown how this ambiguity can be resolved by considering data at varying temperatures. This approach depends on making assumptions about the dynamics of the motions; our approach is entirely kinematic. Sygusch (1976) has shown that, in principle, the ambiguity can also be resolved by a thirdcumulant constrained refinement using Pawley's (1964, 1966) approach, but he also shows that this approach to resolving the ambiguity is of no practical utility.
}

Acta Crystallographica Section B ISSN 0108-7681 $\quad$ C) 1998 
body motion of a molecule. T, $\mathbf{L}$ and $\mathbf{S}$ can be found by a straightforward linear least-squares analysis of the ADP's determined for individual atoms during refinement of the structure, the procedure originally suggested by Cruickshank. Alternatively, they may be determined during structure refinement without the explicit evaluation of individual atomic ADP's, as suggested by Pawley (1964, 1966).

Of course, the rigid-body model is only an approximation at best. The existence of internal molecular motions of fairly large amplitude, particularly internal librations of certain groups, is often apparent from even casual inspection of ORTEP diagrams (Johnson, 1965). Occasional estimates of torsional amplitudes and frequencies of librating groups were being made in the early 1960 's, e.g. for the nitro groups of $p$-nitroaniline (Trueblood et al., 1961) and of $p$-nitrophenol (Coppens \& Schmidt, 1965). Systematic studies in this area were initiated by Johnson $(1967,1970 a)$, with his segmented-body approach, and by Hamilton and his collaborators (Schlemper et al., 1971; Lehmann et al., 1972) in their neutron diffraction investigations of amino acids and other molecules containing methyl groups.

A simple model for the analysis of internal torsional motion from ADP's was introduced by Dunitz \& White (1973). Here, one additional parameter, $\left\langle\varphi^{2}\right\rangle$, the meansquare torsional amplitude of each group suspected of undergoing appreciable torsional motion, is included in the least-squares determination of $\mathbf{T}, \mathbf{L}$ and $\mathbf{S}$. The torsional axis needs to be specified in advance, the group undergoing the torsional motion is assumed to be rigid and all correlations between the internal motion and the overall motion are ignored. This model has been shown (Trueblood, 1978) to yield essentially the same values for mean-square torsional amplitudes in a considerable number of crystals as those derived from more elaborate models for the internal molecular motion, e.g. models including higher cumulants. Moreover, mean-square torsional amplitudes derived from this simple oneparameter model for many groups undergoing torsional motion in crystals are in reasonable agreement with values obtained by other methods (Trueblood \& Dunitz, 1983). There are, however, difficulties that cannot be ignored.

First, there is the problem that the experimental ADP's (which are mean-squared quantities) provide no direct information on the natures, identities or amplitudes of the countless individual modes of motion in the crystal, nor indeed on the relative displacements of different atoms. Hence, in postulating types of internal motion, we must be guided by chemical experience and intuition, and thus may go wrong. Moreover, certain kinds of ambiguity can hardly be avoided. For example, from the ADP's alone it is impossible to distinguish between the torsional motion of a nitro group about the
ONO bisector and the out-of-plane bending of such a group (simulated by torsion about an axis perpendicular to the ONO bisector and in the plane of the group). He \& Craven $(1985,1993)$ have proposed an alternative approach, which they call 'quasinormal mode analysis'. Force constants for a number of presumed lowfrequency internal torsional modes are fitted, together with the rigid-body $\mathbf{T}, \mathbf{L}$ and $\mathbf{S}$, to the observed ADP's. This method also depends on chemical intuition in postulating torsional modes.

A second problem involves the question of correlation among the internal torsional motions and between them and the overall translations and librations. These correlations were ignored by Dunitz \& White (1973). They are addressed here. Bürgi (1989) has considered in detail the correlation of internal translations with overall motion, in an analysis that has many parallels with this one. We did not attempt to include a thirdcumulant constrained refinement, as applied by Prince \& Finger (1973) and by Sygusch (1976) (each ignoring the correlations between overall and internal motion), both because of the increased complexity of the analysis and because we had found that such a refinement gave results not significantly different from those for the Dunitz-White model, even for large libration amplitudes (Trueblood, 1978).

\section{Correlations of internal torsion and overall motion}

The correlations of the torsional motion with the overall molecular motion are analogous to the correlations between overall translation and libration, described by $\mathbf{S}$

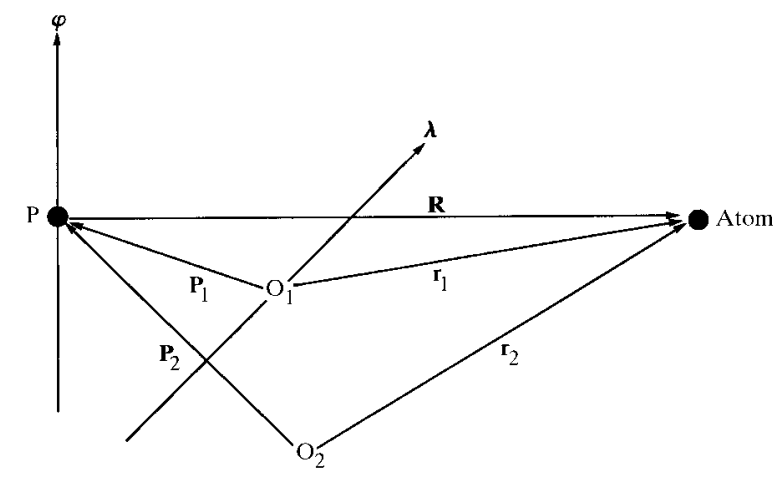

Fig. 1. $\mathrm{O}_{1}$ and $\mathrm{O}_{2}$ are different possible global origins; $\mathbf{r}_{\mathbf{1}}$ and $\mathbf{r}_{\mathbf{2}}$ are vectors from the respective global origins to an atom of interest. If that atom is in an ARG, $\mathbf{R}$ is the vector to that atom from a point, here designated as $\mathbf{P}$, on the axis of the ARG, which is shown as $\boldsymbol{\varphi} . \mathbf{P}_{\mathbf{i}}$ is the vector from the global origin $\mathrm{O}_{\mathrm{i}}$ to $\mathrm{P} . \mathbf{R}=\mathbf{r}_{\mathbf{i}}-\mathbf{P}_{\mathbf{i}}$ is independent of the global origin. The global origin is usually taken to be on an axis of rotation of the molecule $\left(\mathrm{O}_{1}\right)$, but need not be $\left(\mathrm{O}_{2}\right)$. A shift of origin affects $\mathbf{T}$ and $\mathbf{S}$ in predictable ways, but $\mathbf{L}$ is independent of the choice of origin [ST(1968)]. 
in the Schomaker-Trueblood treatment. We limit ourselves here, as earlier, to the quadratic approximation. Consider first a principal rigid body (MAIN) with one non-rigidly attached rigid group (an ARG) that is free to rotate relative to MAIN by the angle $\varphi$ about an axis fixed in MAIN. The total vectorial angle of rotation of the ARG, $\boldsymbol{\Lambda}$, is then equal to the rotation of MAIN, $\lambda$, plus the rotation, $\varphi \equiv \varphi^{\|}$, of the ARG relative to MAIN around the $\operatorname{ARG}(\varphi)$ axis, i.e. $\Lambda=\lambda+\varphi$, the component of rotational displacement, $\Lambda^{\|}$, parallel to the $\varphi$ axis being peculiar to the ARG atoms but the other components, perpendicular to the $\varphi$ axis, being precisely the same for MAIN as for ARG. It is only the total libration about the ARG axis, $\Lambda^{\|} \equiv \lambda^{\|}+\varphi$, that is determinable, not either the mean-square separate amplitude of $\varphi$ or the correlation of $\varphi$ with $\lambda \|$.

If an atom lies on the $\varphi$ axis, its position is not affected by the value of $\varphi$ and we shall not regard it as being a member of the ARG. In general, the ARG axis will not pass through the origin. If $\mathbf{P}$ is the vector from the origin to a point $\mathrm{P}$ on the $\boldsymbol{\varphi}$ axis, the instantaneous displacement of an ARG atom at distance $\mathbf{r}$ from the origin is, in first approximation

$$
\mathbf{u}=\mathbf{t}+\lambda \times \mathbf{P}+\boldsymbol{\Lambda} \times(\mathbf{r}-\mathbf{P}),
$$

$\mathbf{t}$ being the displacement of the origin, $\lambda \times \mathbf{P}$ the displacement of $\mathrm{P}$ relative to the origin and $\boldsymbol{\Lambda} \times(\mathbf{r}-\mathbf{P})$ the displacement of the ARG atom relative to $P$. Letting $\mathbf{t}^{*}=\mathbf{t}+\lambda \times \mathbf{P}$ and $\mathbf{R}=\mathbf{r}-\mathbf{P}$, we rewrite (4) as

$$
\mathbf{u}=\mathbf{t}^{*}+\boldsymbol{\Lambda} \times \mathbf{R} .
$$

For $\mathbf{U}$ we then have

$$
\begin{aligned}
\mathbf{U} \stackrel{\text { def }}{=}\langle\mathbf{u u}\rangle= & \left\langle\mathbf{t}^{*} \mathbf{t}^{*}\right\rangle+\left\langle\mathbf{t}^{*} \boldsymbol{\Lambda}\right\rangle \times \mathbf{R}-\mathbf{R} \times\left\langle\boldsymbol{\Lambda} \mathbf{t}^{*}\right\rangle \\
& -\mathbf{R} \times\langle\boldsymbol{\Lambda} \boldsymbol{\Lambda}\rangle \times \mathbf{R} .
\end{aligned}
$$

Here the terms for $\mathbf{U}$ are separated according to order in $\mathbf{R}$ - zeroth order in the first, first order in the second and third, and second order in the last. Accordingly, if the number of ARG atoms with independent position vectors is sufficiently large, $\langle\mathbf{\Lambda} \mathbf{\Lambda}\rangle,\left\langle\boldsymbol{\Lambda} \mathbf{t}^{*}\right\rangle$ and $\left\langle\mathbf{t}^{*} \mathbf{t}^{*}\right\rangle$ can all be evaluated independently.

As in ST(1968), where we required $\mathbf{u}$ and $\lambda$ (and hence $\mathbf{U}$ and $\mathbf{L}$ ) to be independent of the choice of global origin, so now $\mathbf{u}$ and $\boldsymbol{\Lambda}$ (and hence $\mathbf{U}$ and $\langle\boldsymbol{\Lambda} \mathbf{\Lambda}\rangle$ ) are independent of the choice of global origin. (Note that $\boldsymbol{\Lambda}=\boldsymbol{\lambda}+\boldsymbol{\varphi}$ and we expect each of the latter quantities to be so independent.) On the other hand, $\mathbf{t}^{*}=\mathbf{t}+\lambda \times \mathbf{P}$ is surely dependent on the choice of global origin, since the vector $\mathbf{P}_{\mathbf{i}}$ from the origin $\mathrm{O}_{i}$ to the point $\mathrm{P}$ is (Fig. 1). Hence, the analogs of $\mathbf{T}, \mathbf{S}$ and $\mathbf{S}^{\mathbf{T}}$ in (6) change with a change in global origin [compare (3) and (6)].

Comparison of (5) with (1) or of (6) with (2) or (3) brings out an essential equivalence of ARG to MAIN, if $\boldsymbol{\Lambda}$ and $\mathbf{t}^{*}$ are recognized as the total rotation of the ARG (about the origin) and its translation (at $\mathrm{P}$ ). The equivalence $\uparrow$ of an ARG to a MAIN implies that there is only one quantity with respect to the rotation of an ARG about its axis concerning which inferences can be drawn from the ADP's; this is the total rotation $\Lambda^{\|}$, not the separate rotations $\lambda^{\|}$and $\varphi$. It also shows that for an ARG, again in analogy with $\operatorname{Tr}\{S\}$ for MAIN, $\operatorname{Tr}\left\{\boldsymbol{\Lambda} \mathbf{t}^{*}\right\}$ will be ADP-indeterminate. This is not to say that such quantities are not real nor, unfortunately, that the condition imposed, e.g. $\operatorname{Tr}\{S\}=0$, to relieve the corresponding singularity of the equations for all the derived quantities does not affect the values derived for, in this case, $S_{11}, S_{22}$ and $S_{33}$.

To examine the trace of the tensor $\left\langle\boldsymbol{\Lambda} \mathbf{t}^{*}\right\rangle$, we expand $\boldsymbol{\Lambda}$ and $\mathbf{t}^{*}$

$$
\left\langle\boldsymbol{\Lambda} \mathbf{t}^{*}\right\rangle=\langle\lambda \mathbf{t}\rangle+\langle\lambda \lambda\rangle \times \mathbf{P}+\langle\boldsymbol{\varphi t}\rangle+\langle\boldsymbol{\varphi} \lambda\rangle \times \mathbf{P} .
$$

The trace of (7), we have said, is indeterminate and to stay with ST(1968) we retain for MAIN the condition

$$
\operatorname{Tr}\{S\} \stackrel{\text { def }}{=}\left\langle\lambda_{1} t_{1}\right\rangle+\left\langle\lambda_{2} t_{2}\right\rangle+\left\langle\lambda_{3} t_{3}\right\rangle=0 .
$$

Because $\langle\lambda \lambda\rangle(\stackrel{\text { def }}{=} \mathrm{L})$ is symmetrical, the trace of $(\langle\lambda \lambda\rangle \times \mathrm{P})$ in $(7)$ vanishes identically. (This is readily shown by expanding: $\operatorname{Tr}\{\langle\lambda \lambda\rangle \times \mathbf{P}\}=\lambda_{i} e_{i j k} \lambda_{j} P_{k}$, with $e_{i j k}$ the permutation symbol.) $\ddagger$ The indeterminacy of (7) then implies that $\operatorname{Tr}\left\{\boldsymbol{\Lambda} \mathbf{t}^{*}\right\}$ will be found to be equal to $\operatorname{Tr}\{\langle\boldsymbol{\varphi t}\rangle+\langle\boldsymbol{\varphi} \lambda\rangle \times \mathbf{P}\}$. This last expression is particularly simple in a local axial system (which is defined in Appendix $A$ ):

$$
\left\langle\varphi t^{\|}\right\rangle+\left\langle\varphi \lambda^{\perp 1}\right\rangle P^{\perp 2}-\left\langle\varphi \lambda^{\perp 2}\right\rangle P^{\perp 1} .
$$

Bürgi has pointed out to us that, in general, the last two terms in (7), $\langle\boldsymbol{\varphi} \mathbf{t}\rangle+\langle\boldsymbol{\varphi} \lambda\rangle \times \mathbf{P}$ (or the transpose, $\langle\mathbf{t} \varphi\rangle-\mathbf{P} \times\langle\lambda \boldsymbol{\varphi}\rangle)$, are not always separately determinable, because they depend in just the same fashion on $\mathbf{R}$ $(\stackrel{\text { def }}{=} \mathbf{r}-\mathbf{P})$. In the LOCAL system forms of $\langle\varphi \lambda\rangle \times \mathbf{P}$ that involve $\left\langle\varphi \lambda^{\|}\right\rangle$are not determinable, since $\left\langle\varphi \lambda^{\|}\right\rangle$is not.

As a consequence of $\Lambda=\lambda+\varphi$, (4) can also be written as

$$
\mathbf{u}=\mathbf{t}+\lambda \times \mathbf{r}+\boldsymbol{\varphi} \times(\mathbf{r}-\mathbf{P}),
$$

$\dagger$ This equivalence has been demonstrated with several specific examples, e.g. with the 19 non- $\mathrm{H}$ atoms of adenosine (neutron data at $123 \mathrm{~K}$; Klooster et al., 1991). If the roles of the adenine and ribose moieties are interchanged as MAIN and ARG, the magnitude and s.u. of $2\left\langle\varphi \lambda^{\|}\right\rangle+\left\langle\varphi^{2}\right\rangle$ remain the same, although the sign of the term changes, as anticipated. When the results are expressed in a CCS (Appendix $A$ ), the magnitudes, signs and s.u.'s of the correlations are identical for corresponding pairs of $\langle\varphi \lambda\rangle$ and $\langle\lambda t\rangle$ terms. Most of these correlation terms differ in the local axial systems, because the systems are defined differently for different ARG's (the first-affected atoms being different - see Appendix $A$ ).

$\ddagger e_{i j k}=0$ if any two indices are the same, +1 for a cyclic permutation of the indices and -1 for a noncyclic permutation. In our usage here the summation convention is assumed to hold for repeated indices in a given term: e.g. in (11) below, $e_{i j k} \lambda_{j} P_{k}$ implies that for a given value of $i$ the values of $j$ and $k$ each range from 1 to 3 . 
the form used in Dunitz, Schomaker \& Trueblood (1988). The foregoing equations have to be re-expressed for calculation in a way that makes it convenient to deal with $\lambda$, say, as being always the same object with components expressed in a Cartesian Crystal System (or CCS; see Appendix $A$ ), even if the occurrence of several different ARG axes may require that attention be focussed on components $\lambda^{\|}$or $\lambda^{\perp}$ in diverse directions. We now refer the calculation to a set of Cartesian unit basis vectors $\mathbf{e}_{\mathbf{i}}$ with $\mathbf{e}_{\mathbf{i}} \cdot \mathbf{e}_{\mathbf{j}}=\delta_{i j}$, and represent $\boldsymbol{\varphi}$ as $\boldsymbol{\varphi}=\varphi \mathbf{a}=\varphi a_{i} \mathbf{e}_{i}$ with $\mathbf{a} \cdot \mathbf{a}=a_{i}^{2}=1$, choosing (10) simply because it is the form that has been followed in our programs. With $e_{i j k}$ the permutation symbol, (10) in component form, becomes

$$
u_{i}=t_{i}+e_{i j k}\left(\lambda_{j} r_{k}+\varphi a_{j}\left[r_{k}-P_{k}\right]\right) .
$$

It is helpful toward writing $\langle\mathbf{u u}\rangle$ in component form following (11) first to expand (10), writing $\mathbf{R} \stackrel{\text { def }}{=} \mathbf{r}-\mathbf{P}$,

$$
\mathbf{u}=\mathbf{t}+\varphi \mathbf{a} \times \mathbf{R}+\lambda \times \mathbf{r}=\mathbf{t}-\mathbf{R} \times \mathbf{a} \varphi-\mathbf{r} \times \lambda,
$$

and then to rewrite $\mathbf{U}$ for $\mathrm{ARG}$ atoms as

$$
\begin{aligned}
\langle\mathbf{u u}\rangle= & \langle\mathbf{t t}\rangle+\langle\mathbf{t} \varphi\rangle \mathbf{a} \times \mathbf{R}-\mathbf{R} \times \mathbf{a}\langle\varphi \mathbf{t}\rangle \\
& +\langle\mathbf{t} \lambda\rangle \times \mathbf{r}-\mathbf{r} \times\langle\lambda \mathbf{t}\rangle-\mathbf{r} \times\langle\lambda \varphi\rangle \mathbf{a} \times \mathbf{R} \\
& -\mathbf{R} \times \mathbf{a}\langle\varphi \lambda\rangle \times \mathbf{r}-\mathbf{r} \times\langle\lambda \lambda\rangle \times \mathbf{r} \\
& -\mathbf{R} \times \mathbf{a}\langle\varphi \varphi\rangle \mathbf{a} \times \mathbf{R},
\end{aligned}
$$

and the components as

$$
\begin{aligned}
\left\langle u_{i} u_{l}\right\rangle= & \left\langle t_{i} t_{l}\right\rangle+e_{l m n} a_{m} R_{n}\left\langle t_{i} \varphi\right\rangle-e_{i j k} R_{j} a_{k}\left\langle\varphi t_{l}\right\rangle \\
& +e_{l m n} r_{n}\left\langle t_{i} \lambda_{m}\right\rangle-e_{i j k} r_{j}\left\langle\lambda_{k} t_{l}\right\rangle \\
& -e_{i j k} e_{l m n} r_{j}\left[a_{m} R_{n}\left\langle\lambda_{k} \varphi\right\rangle+r_{n}\left\langle\lambda_{k} \lambda_{m}\right\rangle\right] \\
& -e_{i j k} e_{l m n} R_{j} a_{k}\left[r_{n}\left\langle\varphi \lambda_{m}\right\rangle+a_{m} R_{n}\langle\varphi \varphi\rangle\right] .
\end{aligned}
$$

When using (14) to formulate the observational equations, and then the normal equations for the leastsquares determination of the parameters, an additional constraint is needed, because the equations as written include seven distinct parameters and we know that two of them, $\left\langle\varphi \lambda^{\|}\right\rangle$and $\langle\varphi \varphi\rangle$, cannot be separated. We use the constraint $\left\langle\varphi \lambda_{i}\right\rangle a_{i}=0$, i.e. we set $\left\langle\varphi \lambda^{\|}\right\rangle=0$. Table 1 gives the coefficients of the individual ARG parameters that result from (14). This table is the analog of Table 2 in ST(1968).

\section{Singularities}

Linearly dependent combinations of variables represent, in Johnson's (1970a) words, 'a major numerical problem occasionally encountered in segmented body analysis', leading to singular matrices. Johnson's way out of this problem was to use regression on principal components and the related technique of singular-value decomposition. Use of these methods in analysis of molecular motion has been criticized by Filippini et al. (1973, 1974) if reliable values of $\mathbf{L}$ are wanted, e.g. for correction of intramolecular distances. We have encountered various kinds of singularities in the application of different versions of the program THMA and have not yet succeeded in understanding all of them. We have attempted to warn users to avoid situations that will lead to them and, frequently, we try to provide corrective action in the program for those we do understand. THMA itself makes it easy to recognize singularities, because the calculated standard uncertainties (labeled 'e.s.d.'s' by the program) become unusually large, often orders of magnitude larger than the values of the corresponding parameters.

It is clear why a single-atom ARG leads to a singular matrix when one tries to calculate all six of the correlation terms. Imagine the ARG axis to be along axis $\mathbf{1}$ of the CCS. The torsional libration about that axis makes no contribution to the displacement of the ARG atom parallel to the axis, i.e. to $U^{11}$. Consequently, there are only five $U^{i j}$ values to determine the six ARG parameters and the problem is singular. As detailed in Appendix $B,\left\langle\varphi \lambda_{2}\right\rangle$ is completely indeterminate and only combinations of some of the other parameters will be determinable after suitable constraints have been imposed on the matrix. A two-atom ARG, or any collinear ARG, in a general position also leads to a singular matrix (see Appendix $B$ ).

If there are several independent ARG's on a central MAIN part of a molecule - independent in the sense that they have no common atoms - no correlation between their motion can, in general, be calculated. For the special case $\mathrm{ARG}_{1}-\mathrm{MAIN}-\mathrm{ARG}_{2}$ in which the ARG axes are co-planar (e.g. in the $x, y$ plane), then with sufficient number and disposition of ARG and MAIN atoms, both $\boldsymbol{\Lambda}_{1}$ and $\boldsymbol{\Lambda}_{2}$ (the components of the total ARG rotations parallel to the respective ARG axes) will be strongly correlated with the $z$-component of the rotation of MAIN. Then, clearly, $\left\langle\boldsymbol{\Lambda}_{1} \boldsymbol{\Lambda}_{2}\right\rangle$ will be relatively large, i.e. the two ARG rotations will be strongly correlated.

\section{Tests with and without correlation}

We have made many tests of the effects of including the correlation terms introduced in $\mathbf{2}$ above. Three are summarized in Table 2. The most dramatic example that we have seen is in one of the three polymorphs of dimethyl-3,6-dichloro-2,5-dihydroxyterephthalate; it has been discussed in detail and illustrated by Yang et al. (1989, p. 321). Inclusion of the correlation terms produces a fourfold improvement in the 'agreement index', $w R$, between observed and calculated $U^{i j}$, from about 0.126 to 0.032 . One of the $\langle\varphi \lambda\rangle$ terms is unusually large and its sign (and magnitude) is consistent with the differing effects of the torsional libration, $\varphi$, of the carboxylate ester groups and the overall molecular libration about an axis normal to that libration and in the molecular plane. When that correlation is ignored, 
Table 1. Coefficients of ARG parameters corresponding to (14)

Everything is referred to the Cartesian Crystal System $(\mathrm{CCS}) . \mathbf{a}=$ unit vector along ARG axis $; \mathbf{r}=$ vector from global origin to an atom; $\mathbf{P}=$ vector from global origin to a point on ARG axis; $\mathbf{R}=\mathbf{r}-\mathbf{P} ; \mathbf{V}=\mathbf{a} \times \mathbf{R}$.

$\begin{array}{llllllll} & \langle\varphi \varphi\rangle & \left\langle\varphi \lambda_{1}\right\rangle & \left\langle\varphi \lambda_{2}\right\rangle & \left\langle\varphi \lambda_{3}\right\rangle & \left\langle\varphi t_{1}\right\rangle & \left\langle\varphi t_{2}\right\rangle & \left\langle\varphi t_{3}\right\rangle \\ U^{11} & V_{1}^{2} & & 2 z V_{1} & -2 y V_{1} & 2 V_{1} & \\ U^{22} & V_{2}^{2} & -2 z V_{2} & & 2 x V_{2} & \\ U^{33} & V_{3}^{2} & 2 y V_{3} & -2 x V_{3} & & & 2 V_{3} \\ U^{23} & V_{2} V_{3} & \left(y V_{2}-z V_{3}\right) & -x V_{2} & x V_{3} & V_{3} & V_{2} \\ U^{31} & V_{1} V_{3} & y V_{1} & \left(z V_{3}-x V_{1}\right) & -y V_{3} & V_{3} & V_{1} \\ U^{12} & V_{1} V_{2} & -z V_{1} & z V_{2} & \left(x V_{1}-y V_{2}\right) & V_{2} & V_{1}\end{array}$

there is no good way to account for the differing amplitudes of vibration of the two $\mathrm{O}$ atoms of the ester group normal to the approximate molecular plane. More typically, the reduction in the weighted $R$ for the $U^{i j}$ is of the order $10-30 \%$ when the correlation terms are introduced. The second example in Table 2 is triphenylphosphine oxide at $100 \mathrm{~K}$ (Brock et al., 1985). Each of the three independent phenyl groups was allowed to librate about the $\mathrm{P}-\mathrm{C}$ bond. For this crystal, as for most others for which the unresolvable torsional libration about the ARG axis, $\left(2\left\langle\varphi \lambda^{\|}\right\rangle+\left\langle\varphi^{2}\right\rangle\right)$ is appreciably greater than the overall molecular libration in this direction, $\mathbf{L}^{\|}\left(=\left\langle\lambda^{\|} \lambda^{\|}\right)\right.$, the one-parameter Dunitz-White model gives results for the torsional libration not very different from those given by the sixparameter model that includes the correlation terms.<smiles>COC(=O)c1c(O)c(Cl)c(C(=O)OC)c(O)c1Cl</smiles>

(3)

The third example in Table 2, valine (Schlemper et al., 1971), is based on precise neutron diffraction data and illustrates typical results from ARG's for which the 'affected atoms' are H (or D) atoms. No attempt was made here to correct for the well-known internal vibrations of the $\mathrm{H}$ atoms (although we have a version of THMA that does that, in part), so the mean-square torsional amplitudes of the ARG's are doubtless somewhat exaggerated.

\section{Symmetry}

We have made no complete study, analytical or experimental, of the effect of special site symmetry on the observable ARG parameters. We have, however, considered the following situations, some of which are pertinent to molecules in structures for which $U^{i j}$ are available and have tested some of our conclusions successfully, with both real and synthetic data. The ARG axis might lie along a molecular rotation axis, in a molecular mirror plane, or both. We refer to these symmetries as $\mathbf{2}, \mathbf{3}, \mathbf{4}, \mathbf{6}, \mathbf{v}, \mathbf{2 v}, \mathbf{3 v}, \mathbf{4 v}$ and $\mathbf{6 v}$. We do not consider here mirror planes in the molecular point group that lie at an angle to the ARG axis.

In considering the effect of symmetry on the ARG parameters it is important to remember that a rotation is an axial vector, so the effects of symmetry operations on a rotation will sometimes be different from those on a translation parallel to the rotation axis. Consider, for example, a twofold axis and a mirror plane:

$\begin{array}{ccc}\mathbf{t} & \text { Parallel to } \mathbf{2} & \text { Unchanged } \\ & \text { In mirror plane } & \text { Unchanged } \\ & \text { Axis parallel to } \mathbf{2} & \text { Unchanged } \\ & \text { Axis in } \mathbf{m} & \text { Reversed } \\ \mathbf{t} & \text { Perpendicular to } \mathbf{2} & \text { Reversed } \\ & \text { Perpendicular to } \mathbf{m} & \text { Reversed } \\ \boldsymbol{\lambda}, \boldsymbol{\varphi} & \text { Axis perpendicular to } \mathbf{2} & \text { Reversed } \\ & \text { Axis perpendicular to } \mathbf{m} & \text { Unchanged }\end{array}$

Table 3 summarizes our conclusions regarding ARG site symmetry, expressed in the local axial system. Many of the parameters vanish; similar constraints appear when the parameters are expressed in the CCS.

\section{Computer programs}

The computer program used in applying the analysis described here has evolved over the last dozen years. The current version is $T H M A 14 c$. It does all calculations in the CCS and gives the ARG parameters both in the CCS and in the local axial system (Appendix $A$ ) for that ARG, since some results are easier to appreciate, and 
Table 2. Comparison of results with and without correlations

Units are $\mathrm{deg}^{2}$. Correlations of $\varphi$ with $\mathbf{t}$ ignored here.

\begin{tabular}{|c|c|c|c|c|c|}
\hline Structure & ARG & $\left\langle\varphi^{2}\right\rangle_{D W}$ & $2\left\langle\varphi \lambda^{\|}\right\rangle+\left\langle\varphi^{2}\right\rangle$ & $\left\langle\varphi \lambda^{\perp 1}\right\rangle$ & $\left\langle\varphi \lambda^{\perp 2}\right\rangle$ \\
\hline (1) & $-\mathrm{COOCH}_{3}$ & $131(38)$ & $153(13)$ & $0(4)$ & $16(7)$ \\
\hline \multirow[t]{3}{*}{ (2) } & $-\mathrm{C}_{6} \mathrm{H}_{5}(A)$ & $42(5)$ & $41(4)$ & $4(2)$ & $-1(2)$ \\
\hline & $-\mathrm{C}_{6} \mathrm{H}_{5}(B)$ & $5(4)$ & $5(3)$ & $-3(2)$ & $0(2)$ \\
\hline & $-\mathrm{C}_{6} \mathrm{H}_{5}(C)$ & $6(4)$ & $11(3)$ & $1(2)$ & $-1(2)$ \\
\hline \multirow[t]{3}{*}{ (3) } & $-\mathrm{CH}_{3}(A)$ & $250(40)$ & $278(38)$ & $-16(39)$ & $9(32)$ \\
\hline & $-\mathrm{CH}_{3}(B)$ & $212(36)$ & $215(33)$ & $7(33)$ & $1(30)$ \\
\hline & $-\mathrm{NH}_{3}{ }^{+}$ & $54(21)$ & $50(19)$ & $4(18)$ & $-6(20)$ \\
\hline
\end{tabular}

Structure (1): Dimethyl 3,6-dichloro-2,5-dihydroxyterephthalate (Yang et al., 1989). Three atoms (O, O, C) in ARG. wR( $U$ ) decreased from 0.126 to 0.032 when correlations were included. The $\left\langle\varphi \lambda^{\perp 2}\right\rangle$ term affects the two ester O atoms differently. Structure (2): Triphenlphosphine oxide at $100 \mathrm{~K}$ (Brock et al., 1985). Five C atoms in each ARG. $w R(U)$ decreased from 0.107 to 0.075 when correlations were included. Structure (3): Valine (neutron data at $296 \mathrm{~K}$; Schlemper et al., 1971). Three H atoms in each ARG. $w R(U)$ decreased from 0.152 to 0.126 when correlations were included.

compare, in the local system. The program accommodates up to seven different ARG groups, estimates corrections to intramolecular distances $\dagger$ arising from overall and internal motion, and has many options. It can, of course, also handle rigid-body (TLS) calculations, even if no ARG's are present. There is a version designed primarily for handling structures with ADP's for $\mathrm{H}$ and $\mathrm{D}$ atoms (THMA13); it has not been tested as extensively. THMA14c, together with detailed instructions and sample data, is available by ftp from Emily Maverick (maverick@chem.ucla.edu).

We have also written a program to generate U's for a model structure with orthogonal axes, up to 30 atoms at any positions, and up to two ARG's, given any specified input values for $\mathbf{T}, \mathbf{L}, \mathbf{S}, 2\left\langle\varphi \lambda^{\|}\right\rangle+\left\langle\varphi^{2}\right\rangle$ and the correlations $\langle\varphi \lambda\rangle,\langle\varphi \mathbf{t}\rangle$ and $\left\langle\varphi \varphi^{\prime}\right\rangle$. The structural parameters are then analyzed by THMA to see whether we get back the values for $\mathbf{T}, \mathbf{L}, \mathbf{S}$ and the various $A R G$ parameters that we input initially. This program has helped in clearing up some problems (with both our understanding and the program THMA) and we believe that it provides one of the best approaches to reaching generalizations about various kinds of singularities - but much is still murky.

\section{APPENDIX $A$ \\ Axial systems}

Various axial systems are alluded to here and in the computer programs referred to. Let the crystal axes be $\mathbf{a}_{\mathbf{i}}$. The CCS (Cartesian Crystal System) that was used in ST(1968) and also here, as well as in our programs, has axes parallel to $\mathbf{a}_{1}, \mathbf{a}_{3} \times \mathbf{a}_{1}$ and in a direction normal to the first two, in a right-handed sense. We also use a Cartesian local axial system in considering the motion of a given ARG. This local system is unique to each ARG.

$\dagger$ Most of these corrections have been admirably discussed by Johnson $(1970 b)$. We also include estimates of corrections for anharmonicity of $X-\mathrm{H}$ and $X-\mathrm{D}$ bonds, which are usually negative, and may offset the usual riding corrections (Ibers, 1959; Craven \& Swaminathan, 1984; Jeffrey \& Ruble, 1984).
The torsional axis (the direction of $\varphi$ ) is designated by $"$. The other two axes are chosen so as to form a righthanded system with the first axis. The second axis, designated here by ${ }^{\perp 1}$, is in the direction of motion of the first-listed atom that is affected by the motion of the ARG about the $\varphi$ axis; it is thus necessarily normal to the $\varphi$ axis. This definition, while arbitrary, has proved convenient. The third axis, designated by ${ }^{\perp 2}$, is normal to the first two. Other reasonable definitions of local axial systems are possible; that just described has been used in all of our computer programs.

\section{APPENDIX $B$}

B1. Proofs concerning singularities of two-atom and oneatom $A R G$ 's

B1.1. Two-atom ARG, or any multi-atom collinear $A R G$, not lying on a symmetry element. General case: vector between the ARG atoms not parallel to the ARG axis. Define a vector $\Delta \mathbf{r}$ between any of the collinear atoms of the ARG. Without loss of generality, take axis $\mathbf{1}$ to be the ARG axis a (the direction of $\varphi$ ); take axis $\mathbf{3}$ parallel to $\mathbf{a} \times \Delta \mathbf{r}$, and let it intersect both $\mathbf{a}$ and $\Delta \mathbf{r}$ to define the origin. Axis $\mathbf{2}$ is normal to the other two axes in a right-handed sense (Fig. 2).

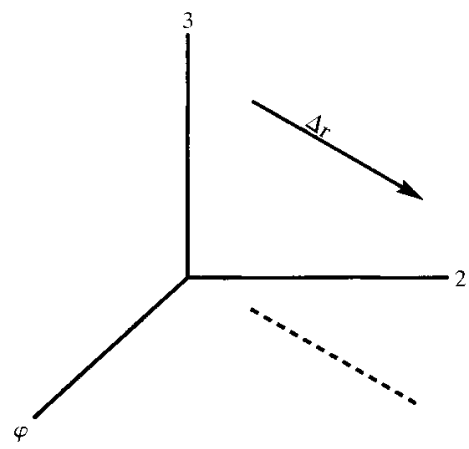

Fig. 2. The two-atom ARG, lying along $\Delta \mathbf{r}$. The broken line is the projection of $\Delta \mathbf{r}$ on the $x, y$ plane. The libration axis of the ARG is $\mathbf{1}$ (the $\varphi$ axis). 
Table 3. Effect of symmetry on ARG parameters

' 0 ' implies term must vanish; ' $x$ ' implies term is unrestricted.

$\begin{array}{lllllll}\text { Symmetry } & 2\left\langle\varphi \lambda^{\|}\right\rangle+\left\langle\varphi^{2}\right\rangle & \left\langle\varphi \lambda^{\perp 1}\right\rangle & \left\langle\varphi \lambda^{\perp 2}\right\rangle & \left\langle\varphi t^{\|}\right\rangle & \left\langle\varphi t^{\perp 1}\right\rangle & \left\langle\varphi t^{\perp 2}\right\rangle \\ \mathbf{2} & x & 0 & 0 & x & 0 & 0 \\ \mathbf{3 , 4 , 6} & x & 0 & 0 & x & 0 & 0 \\ \mathbf{v} & x & 0 & x & 0 & x & 0 \\ \mathbf{n v} & x & 0 & 0 & 0 & 0 & 0\end{array}$

Since the origin is on the ARG axis, $\mathbf{P}=0$ and $\mathbf{r}=\mathbf{R}$, and since axis $\mathbf{1}$ is the ARG axis, $a_{1}=1$, and $a_{2}=a_{3}=0$. The vector $\Delta \mathbf{r}$ passes through axis $\mathbf{3}$; consequently, the $x$ and $y$ coordinates of the atoms of the ARG are related by $x_{i}=k y_{i}$. Furthermore, since the vector $\Delta \mathbf{r}$ is normal to axis $\mathbf{3}$, all atoms of the ARG have the same coordinate $z_{o}$. Under these circumstances, $V_{1}=0, V_{2}=-z_{o}$, and $V_{3}=y$. Table 1 then simplifies to Table 4.

This leads at once to the familiar singularity involving $\langle\varphi \varphi\rangle$ and $\left\langle\varphi \lambda_{1}\right\rangle$ (which is here the same as $\left\langle\varphi \lambda^{\|}\right\rangle$), with all the terms in the column for the second of these quantities being just twice those for the first. The singularity has been allowed for by the constraint on the matrix alluded to just after (14). A second singularity becomes evident when the coefficients of columns 2, 3, 5 and 6 are considered, and $k y$ is substituted for $x$. The following combination is then found to add to zero, which implies that the matrix of normal equations will be singular

$$
\begin{aligned}
& k(\text { column } 2)+\text { column } 3-z_{o}(\text { column } 5) \\
& +k z_{o}(\text { column } 6)=0
\end{aligned}
$$

Special case: $\boldsymbol{\Delta} \mathbf{r}$ is parallel to $\mathbf{a}$ (axis $\mathbf{1}$ ). Choose axis $\mathbf{2}$ perpendicular to the ARG axis $\mathbf{a}$ and in the plane of $\Delta \mathbf{r}$ and $\mathbf{a}$; axis $\mathbf{3}$ is normal to the first two axes, in a righthanded sense. All ARG atoms have $z=0$ and have the same $y$ coordinate, $y_{o}$, but different values of $x$. Substitution into Table 4 above again shows two singularities. The quantities that can be determined are $\left\langle\varphi \lambda_{2}\right\rangle,\left\langle\varphi \lambda_{3}\right\rangle,\left\langle\varphi t_{1}\right\rangle,\left\langle\varphi t_{2}\right\rangle$ and the combination $y_{o}\left(\langle\varphi \varphi\rangle+2\left\langle\varphi \lambda_{1}\right\rangle\right)+2\left\langle\varphi t_{3}\right\rangle$.

B1.2. Single-atom ARG. Without loss of generality, take axis 1 to be the ARG libration axis a (the direction of $\varphi$ ) and the global origin to be on the ARG axis, at the point where the normal from the ARG atom (axis 2) hits the ARG axis. Axis 3 is normal to the other two axes, in a right-handed sense. Since the origin is on the ARG axis, $\mathbf{P}=0$ and $\mathbf{r}=\mathbf{R}$, and since axis $\mathbf{1}$ is the ARG axis, $a_{1}=1$ and $a_{2}=a_{3}=0$. The only nonzero coordinate of the ARG atom is $y_{o}$. Substitution of these conditions into Table 4 shows that $\left\langle\varphi \lambda_{2}\right\rangle$ is indeterminate and that only three quantities are determinable, if suitable constraints on the matrix are imposed: $y_{o}\left\langle\varphi \lambda_{2}\right\rangle-\left\langle\varphi t_{1}\right\rangle$, $\left\langle\varphi t_{2}\right\rangle$ and $\left[y_{o}\left(\langle\varphi \varphi\rangle+2\left\langle\varphi \lambda_{1}\right\rangle\right)+2\left\langle\varphi t_{3}\right\rangle\right]$.
Table 4.

$\begin{array}{llllllll} & \langle\varphi \varphi\rangle & \left\langle\varphi \lambda_{1}\right\rangle & \left\langle\varphi \lambda_{2}\right\rangle & \left\langle\varphi \lambda_{3}\right\rangle & \left\langle\varphi t_{1}\right\rangle & \left\langle\varphi t_{2}\right\rangle & \left\langle\varphi t_{3}\right\rangle \\ U^{11} & & & & & & & \\ U^{22} & z_{o}^{2} & 2 z_{o}^{2} & & -2 x z_{o} & & -2 z_{o} & \\ U^{33} & y^{2} & 2 y^{2} & -2 x y & & & & 2 y \\ U^{23} & -y z_{o} & -2 y z_{o} & x z_{o} & x y & & y & -z_{o} \\ U^{31} & & & y z_{o} & -y^{2} & y & & \\ U^{12} & & & -z_{o}^{2} & y z_{o} & -z_{o} & & \end{array}$

We have benefitted from many discussions with HansBeat Bürgi, who provided the proofs in Appendix $B$, and with Jack Dunitz and Emily Maverick, and from the helpful suggestions of Uri Shmueli and several referees.

\section{References}

Brock, C. P., Schweizer, B. \& Dunitz, J. D. (1985). J. Am. Chem. Soc. 107, 6964-6970.

Bürgi, H. B. (1989). Acta Cryst. B45, 383-390.

Bürgi, H. B. (1995). Acta Cryst. B51, 571-579.

Coppens, P. \& Schmidt, G. M. J. (1965). Acta Cryst. 18, 654-663.

Craven, B. M. \& Swaminathan, S. (1984). Trans. Am. Cryst. Assoc. 20, 133-135.

Cruickshank, D. W. J. (1956). Acta Cryst. 9, 754-756.

Dunitz, J. D., Maverick, E. F. \& Trueblood, K. N. (1988). Angew. Chem. Int. Ed. Engl. 27, 880-895.

Dunitz, J. D., Schomaker, V. \& Trueblood, K. N. (1988). J. Phys. Chem. 92, 856-867.

Dunitz, J. D. \& White, D. N. J. (1973). Acta Cryst. A29, 93-94.

Filippini, G., Gramaccioli, C. M., Simonetta, M. \& Suffritti, G. B. (1973). J. Chem. Phys. 59, 5088-5101.

Filippini, G., Gramaccioli, C. M., Simonetta, M. \& Suffritti, G. B. (1974). Acta Cryst. A30, 189-196.

Förtsch, M. (1997). Ph.D. Dissertation. University of Bern.

He, X. \& Craven, B. M. (1985). Acta Cryst. A41, 244-251.

He, X. \& Craven, B. M. (1993). Acta Cryst. A49, 10-22.

Ibers, J. A. (1959). Acta Cryst. 12, 251-252.

Jeffrey, G. A. \& Ruble, J. R. (1984). Trans. Am. Cryst. Assoc. 20, 139-142.

Johnson, C. K. (1965). ORTEP. Report ORNL-3794. Oak Ridge National Laboratory, Tennessee, USA.

Johnson, C. K. (1967). Abstr. Amer. Crystallog. Assoc. Summer Meeting, Minneapolis, p. 82.

Johnson, C. K. (1970a). Thermal Neutron Diffraction, edited by B. T. M. Willis, pp. 132-160. Oxford University Press.

Johnson, C. K. (1970b). Crystallographic Computing, edited by F. R. Ahmed, pp. 220-226. Copenhagen: Munksgaard.

Klooster, W. T., Ruble, J. R., Craven, B. M. \& McMullan, R. K. (1991). Acta Cryst. B47, 376-383.

Lehmann, M. S., Koetzle, T. F. \& Hamilton, W. C. (1972). J. Am. Chem. Soc. 94, 2657-2660.

Pawley, G. S. (1964). Acta Cryst. 17, 457-458.

Pawley, G. S. (1966). Acta Cryst. 20, 631-638.

Prince, E. \& Finger, L. W. (1973). Acta Cryst. B29, 179-183.

Schlemper, E. O., Hamilton, W. C. \& LaPlaca, S. J. (1971). J.

Chem. Phys. 54, 3990-4000. 
Schomaker, V. \& Trueblood, K. N. (1968). Acta Cryst. B24, 63- Trueblood, K. N. \& Dunitz, J. D. (1983). Acta Cryst. B39, 12076. 133.

Schomaker, V. \& Trueblood, K. N. (1984). Acta Cryst. A40, C- Trueblood, K. N., Goldish, E. \& Donohue, J. (1961). Acta Cryst. 339. 14, 1009-1017.

Sygusch, J. (1976). Acta Cryst. B32, 3295-3298.

Trueblood, K. N. (1978). Acta Cryst. A34, 950-954.

Yang, Q.-C., Richardson, M. F. \& Dunitz, J. D. (1989). Acta Cryst. B45, 312-323. 